\title{
ANALYSIS OF CORE-VALUES ALIGNMENT IN COLLABORATIVE NETWORKS
}

\author{
Luis M. Camarinha-Matos \\ New University of Lisbon, Quinta da Torre - 2829 Monte Caparica, PORTUGAL \\ cam@uninova.pt \\ Patrícia Macedo \\ Escola Superior de Tecnologia de Setúbal, Instituto Politécnico de Setúbal, PORTUGAL \\ pmacedo@est.ips.pt \\ António Abreu \\ New University of Lisbon, Quinta da Torre - 2829 Monte Caparica, PORTUGAL \\ ajfa@fct.unl.pt

\begin{abstract}
The identification and characterization of core-values in collaborative networks is an important element for the identification of a potential for conflicts. This paper introduces an approach, based on causal models and graph theory, for the analysis of core-values alignment in networked organisations. The potential application of this approach is also discussed in the VO breeding environment context.
\end{abstract}

\section{INTRODUCTION}

Most works on Collaborative Networks (CNs) are focused on the (potential) benefits of collaboration. However, participation in a $\mathrm{CN}$ also involves risks and often consortia fail due to internal conflicts. Conflicts can be originated by different corevalues priorities and different perceptions of outcomes. The perception of outcomes is to some extent subjective in the way that it depends of the preferences of the subject and how exchanges are evaluated. The set of core-values and preferences hold by an individual or organization is defined in its value system.

In a collaborative network environment the value system of each network member may influence the success of collaboration. Therefore, when considering a candidate to join a network, it is important to assess this potential member according to the set of core-values that it holds and the network's core-values. Such analysis should provide elements that help managers to detect a potential for conflicts or the likelihood of the new member contributing to add value to the network.

Values in organizations have been studied during the last decades using diverse approaches that are focused essentially on four aspects: creation of organizational core-values taxonomies; development of methodologies to collect organizational core-values; development of frameworks in order to classify organizations according to their core-values; and analysis of the relation between the core-values held by employees and organizational core-values. For instance, the social researchers 
Rokeach and Schwartz (Rokeach, 1973; Schwartz, 1992) developed some empirical work to identify core-values. Based on this work they proposed organizational corevalues taxonomies. Brian Hall and Richard Barret (Barrett, 2006; Hall, 1995) developed theories about values in organizations, and discussed the importance of values management for the success of organizations. Their works contributed as well to clarify the differences between the core-values hold by organizations and their expected core-values. Richard Barret also studied the alignment between employee's core-values and enterprise's core-values. On the other hand, Eden (Eden, 1992) used causal maps to represent the cognitive structure of core-values. This work establishes the relationships between organizational goals and core-values. Another cognitive approach was proposed by Rekom and his colleagues (Rekom, Riel and Wierenga, 2006) as a methodology to measure core-values based on daily actions.

In recent years some studies have explored the importance of value systems in the context of networked organizations (Abreu and Camarinha-Matos, 2006; Afsarmanesh and Camarinha-Matos, 2005; Macedo, Sapateiro and Filipe, 2006; Zineldin, 1998), however none of them proposed methodologies, approaches or support tools to help network managers to analyze Virtual Organization's (VO) Value System in a Virtual organizations Breeding Environments (VBE) context.

The aim of this paper is to propose an approach based on graph theory and causal maps to analyze the core-values alignment in collaborative networked environments. This work aims at contributing to answer the following main questions:

- How to identify which values can a potential new member add to the VBE core-values?

- How to analyze the alignment between VBE core-values and the set of corevalues of a potential new member?

- How to analyze the potential for conflicts among VO members?

- How to analyze the alignment between VO's core-values and VO members' core-values?

\section{VALUE SYSTEM BASE CONCEPTS}

There is no consensus about the value system definitions among the various disciplines (e.g. economy, sociology, artificial intelligence) that have addressed this topic. In an attempt to provide a "unified" definition, (Camarinha-Matos and Macedo, 2007) proposed a conceptual model for value systems embracing the economic and sociological notions. In order to discuss the core-value concept it is necessary to first summarize the generic conceptual model of value system introduced in previous work (Camarinha-Matos, Macedo, 2007).

This conceptual model identifies as main elements of a value system:

- Object of Evaluation - Something $(x)$ that can be evaluated, and have value for the evaluator:

$x \in S$ where: $\mathrm{S}$ is the set of things that can be evaluated.

- Evaluation Functions - The functions used to implement an evaluation act.

$f \in F: F=N F \cup Q F$, where NF is the set of numeric functions, and $\mathrm{QF}$ the set of qualitative functions. 
- Evaluation Dimensions - Characteristics of an object that are evaluated. $D=\left\{d_{1}, d_{2}, \ldots d_{n}\right\} \quad$ is the set of evaluation dimensions. Furthermore $f \Phi d$ means: the function $f$ permits to evaluate the dimension $d$.

- Evaluation Perspective - A selected set of evaluation dimensions and the corresponding weights chosen to evaluate an object from a given point of view.

$e p_{x}=<d v_{x}, w v>\in \mathrm{P}$, where $x \in S$ and $\mathrm{P}$ is the set of evaluation perspectives.

$w v$ represents the weights-vector and $d v_{x}$ expresses the set of dimensions of an object that is evaluated (dimensions-vector), where:

$$
d v_{x}=\left[d_{1}, d_{2}, \ldots . d_{n}\right]: d_{i} \in D \quad w v=\left[w_{1}, \ldots . w_{n}\right]: w_{i} \in[0 . .1] \wedge \sum_{i=0}^{n} w_{i}=1
$$

i.e., $w v[i]$ represents the degree of importance of the characteristic $d v_{x}[i]$

For each dimensions-vector an evaluation-vector can be specified as:

$$
f v_{d}=\left[f_{1}, f_{2}, \ldots . f_{n}\right]: f_{i} \in F \text {, where } i \in[1 . . n] \wedge f v_{d}[i] \Phi d v_{x}[i]
$$

In order to represent the fact that an object can be evaluated through different perspectives, the operator $\Xi$ is defined as: $x \Xi e p$, meaning $\mathbf{x}$ is evaluated through the perspective $e p$, where $x \in S \wedge e p \in P$.

These elements are further organized in two sub-groups:

- Entities that can be evaluated: Objects of evaluation.

- Evaluation mechanism: Functions, Dimensions, and Perspectives.

Based on these elements, a Value System is thus composed of a set of valuable things for an organization and a set of functions used for its evaluation according to different perspectives, where each perspective is composed of a weighted set of evaluation dimensions.

Value System - VS $=<E V S, R V S>$ where $E V S=<O S, E S>$ is the aggregation of the two subsystems that compose the value system and $R V S$ represents the set of relationships between the two sub-systems:

- Value Objects Subsystem - OS $=<\mathrm{S}, \mathrm{RS}>$ where: $S$ is the set of valuable things; RS is the set of relationships among the elements of $\mathrm{S}$.

- Evaluation Subsystem - $E S=<E F, R E>$ where: $E F$ is defined as a triple: $E F=<F, D, P>F$ is the set of evaluation functions; $D$ is the set $o$ evaluation dimensions; $P$ is the set of evaluation perspectives; and $R E$ is the set of relationships among the elements of $E F$.

Each organization (or network of organizations) considers a set of characteristics as the most important for itself; these characteristics are called core-values (Collins and Porras, 1996). The core-values are used as the base for the decision-making processes and they are the elements that motivate and regulate its own behaviour (Hall, 1995; Higgins, 2004). Therefore we can introduce the notion of Core Value System to encompass the core-values. This concept is a restricted view of the 
generic value system model presented above, and can be considered as specialization of it.

This model assumes that core-values are the core characteristics of the organisation (or network of organizations) to be evaluated. Thus, a core-value is defined as an evaluation dimension of the Core Value System and the organisation (or network of organizations) as the (sole) object of evaluation (see Figure 1).

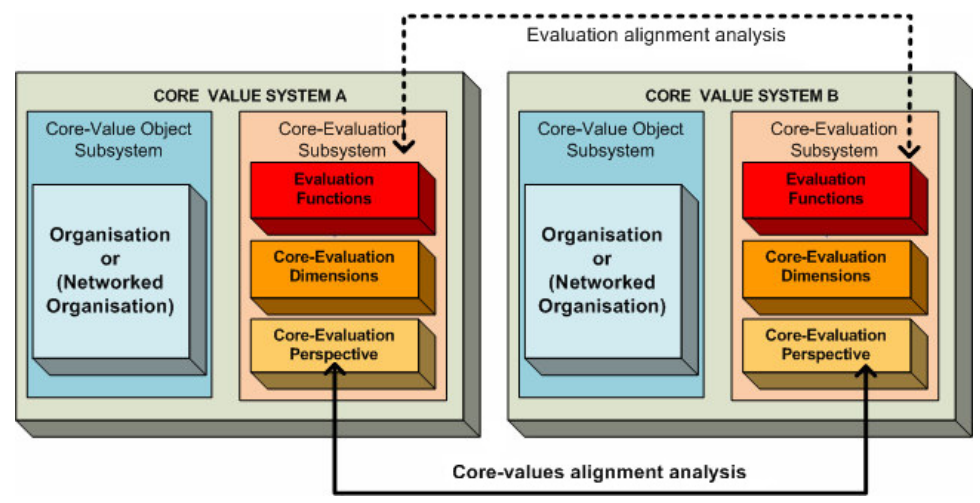

Figure 1 - Core Value System components and alignment analysis.

Core-Value System - $C V S=<C E V S, C R V S>$ where $C E V S=<C O S, C E S>$ is the aggregation of the two subsystems that compose the core-value system and $C R V S$ represents the set of relationships between the two sub-systems:

- Core Value Objects Subsystem (COS) is represented by the organisation (or networked organisation) itself.

- Core Evaluation Subsystem - CES $=<C E F, C R E>$ where: CEF is defined as a triple: $C E F=<C F, C V, C P>C F$ is the set of evaluation functions to evaluate the organisation core-values; $C V$ is the set of core-values; $C P$ is the core-evaluation perspective; and $C R E$ is the set of relationships among the elements of $C E F$.

The core-evaluation perspective is defined as:

$$
e p_{\text {core }}=<d v_{\text {core }}, w v_{\text {core }}>\in C P \text {, where: }
$$

- $\quad d v_{\text {core }}$ expresses the vector of core-values of the organisation.

- $w v_{\text {core }}$ represents the weights -vector, where each element defines the degree of importance of the respective core-value. These weights represent the preferences of the value-system's owner.

Example: Let us suppose the core-values held by a Logistic Enterprise are innovation, reliability, and profit and each core-value has a different degree of importance (see Figure 2). This set of core-values is thus part of the Core Value System, and more specifically part of the core-evaluation subsystem (CES).

$C V=\{$ innovation, profit, reliability $\} \in C E S$ 
- $\quad d v_{\text {core }}=$ [innovation, profit, reliability]

- $w v_{\text {core }}=[0.1,0.5,0.4]$, representing the enterprise 's preferences.

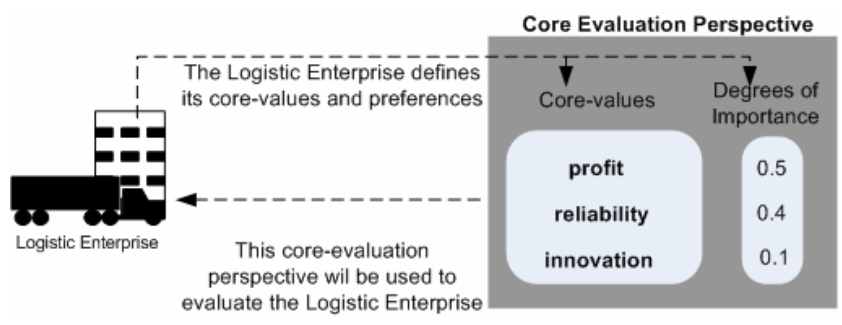

Figure 2- Core values and core evaluation perspective concepts.

According to this approach, the analysis of the alignment between two Core Value Systems has to comprise two main aspects (see Figure 2):

- The analysis of core-values alignment, where the compatibility between the two sets of core-values is analyzed.

- The analysis of evaluation alignment, where it is analyzed whether the evaluation functions of different Core Value Systems, used to evaluate the same characteristic, are similar. Although, two evaluators could hold the same core-value, if they use different evaluation functions, the evaluation results could be distinct.

However, the aim of this paper is to solely discuss the core-values alignment.

\section{A META-MODEL TO ANALYZE CORE-VALUES}

In order to analyze the core-values of a collaborative network it is necessary to have a model that supports the analysis of the relationships among the following entities: core-values, organizations, and VOs. Therefore, as a first approach, let us consider the following relationships:

1. Core-values and core-values - in order to understand how core-values influence each other.

2. Core-values and organisations - in order to know which core-values are held by each organization.

3. Core-values and VOs - in order to understand which core-values are held by the VO.

These relationships can be modelled using graphs. The main goal is to represent a network in symbolic terms, abstracting reality as a set of linked nodes. In this case each node represents an element (a VO, an organization, or a core-value) and the directed arcs specify the relationships. On the other hand, an extension of graph theory is the Causal Models that naturally emerged due to the need for a sketching technique to support and facilitate reasoning about cause and effect. Causal modelling builds upon a binary relationship, called an influence relationship, between two entities that represent named quantitative or qualitative values or value sets. Whereby changes in the influencing entity are conveyed as changes in the influenced entity (Greenland and Brumback, 2002). In this case, the causal 
modelling method is used to model the causal relations among core-values in order to analyze the influence among them.

Considering the nature of this analysis, as first approach, a combination of these two modelling techniques is suggested, as illustrated in Table 1

Table 1 - Framework to analyze core-values in CNO's.

\begin{tabular}{|c|c|c|c|}
\hline & Core-Values & Organization & VO \\
\hline \multirow{4}{*}{ 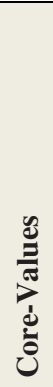 } & Core-values influence map. & $\begin{array}{l}\text { Organization's } \\
\text { core-values map }\end{array}$ & VO's core-values map \\
\hline & $\begin{array}{l}\text { Use causal maps to show how } \\
\text { core-values influence positively } \\
\text { or negatively each other. }\end{array}$ & $\begin{array}{l}\text { Use graphs to show the } \\
\text { core-values held by each } \\
\text { organisation. }\end{array}$ & $\begin{array}{l}\text { Use graphs to show the } \\
\text { core-values held by the } \\
\text { VO., and the core-values } \\
\text { shared by VOs. }\end{array}$ \\
\hline & Positive influence relationship & Organisation $\mathrm{A}$ & $\stackrel{\text { voi }}{\longrightarrow}$ \\
\hline & core-value $\mathrm{x}$ & Ownership relation & Ownership relation \\
\hline & Negative influence relationship & & \\
\hline
\end{tabular}

\section{POTENTIAL APPLICATION}

In order to detect a potential for conflicts and to promote alignment between (core) Value Systems, this example illustrates how the proposed approach can be used to answer the research questions introduced above. For that, let us assume the existence of a Reference Core-Values Knowledge Base, which contains a description about every possible core-value that an organisation can hold. This knowledge base also stores the information about the influence relationship between pairs of corevalues. This knowledge can be provided by experts or result of surveys and interviews (see (Rekom, 2006) and (Hall, 1995), as examples). The creation of a Reference Core-Values Knowledge Base has two main purposes:

- To allow that the selection of core-values is done from a limited set. This will guarantee the existence of a common terminology.

- To allow that the generation of core-values maps from a subset of corevalues is done consistently. This will permit to compare core-value maps of network members and to analyze the influence among core-values of distinct members.

In the proposed example scenario, the existence of a $V O$ breeding environment (VBE) is considered which initially contains seven organizations: a bank, two universities, three factories and one logistics operator, as illustrated in Figure 3. 


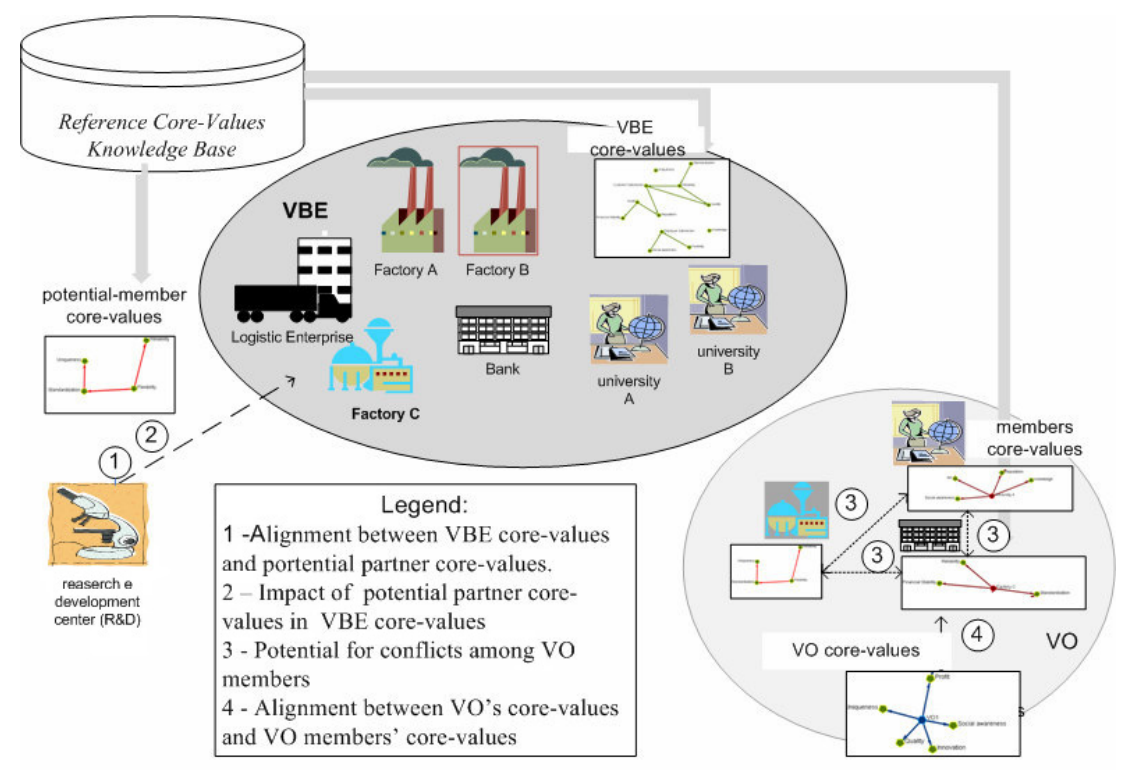

Figure 3 - Example VBE scenario.

In this context, when a VBE is formed, each member can select from the preconfigured list of reference core-values, the ones that it considers as its core-values. The pre-configured list is generated from the data stored in the Reference CoreValues Knowledge Base. From these data the following maps can be generated:

1. Core-values map. This causal map shows the influences between VBE's corevalues, as illustrated in Figure 4 .

2. Organisation's core-values map. This two-mode graph shows both the corevalues held by VBE members and how they are shared, as illustrated in Figure 5.

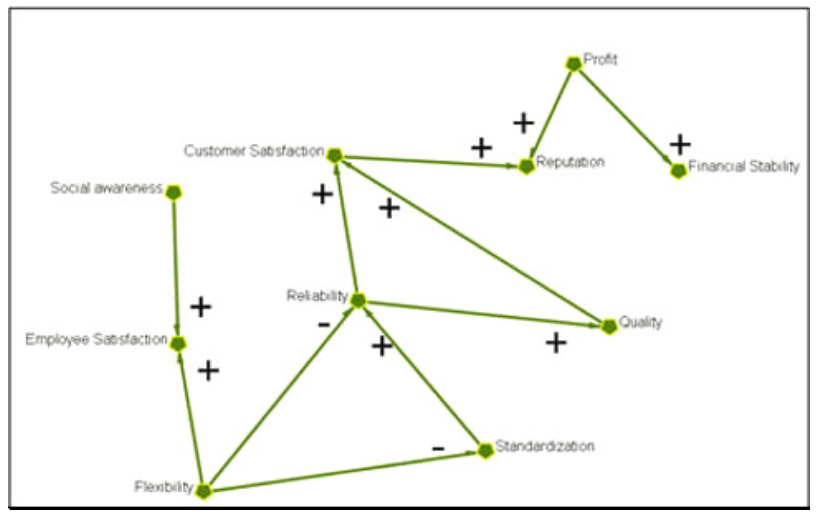

Figure 4 - Core-values influence map 


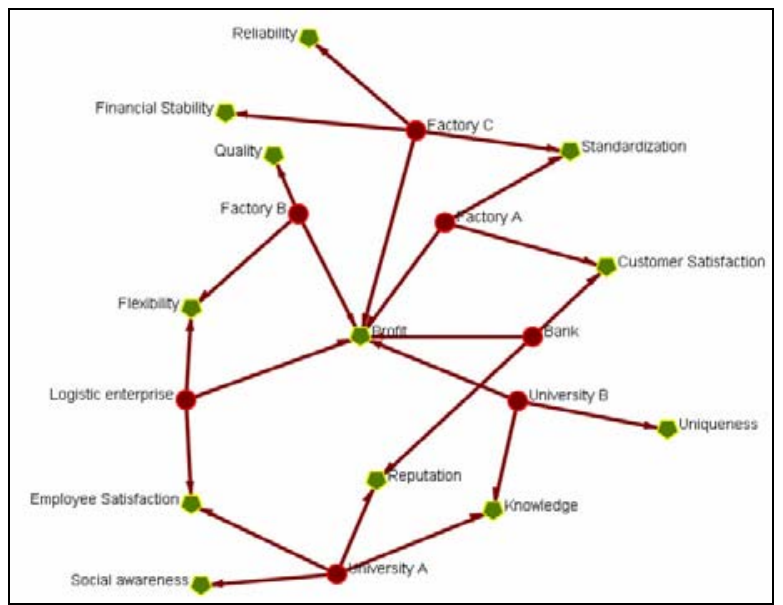

Figure 5 - Initial Organization's core-values map

Furthermore, in order to analyze the alignment between VBE core-values and the set of core-values of a potential partner, let us suppose that the Research and Development Center (RDC) wants to join the VBE and Innovation, Knowledge, and Uniqueness are its core-values.

In order to analyze the alignment between the core-values of the VBE and the core-values of the Research Center and to identify which values can be added to the VBE core-values, a causal map is generated, as shown in Figure 5. The comparative analysis of these two causal maps (Figure 5 and Figure 6) shows that Innovation was added to the map and that it can influence negatively the Standardization. This means that there exists a potential for conflicts between the RDC and other VBE members that have Standardization as a core-value.

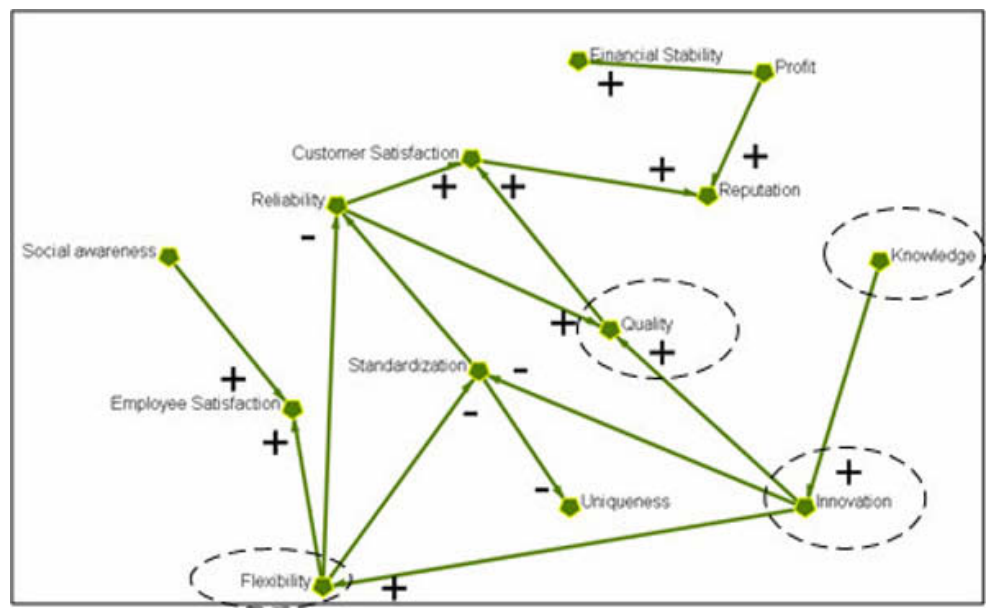

Figure 6 - Core-values influence map after RDC joined the VBE. 
On the other hand, the analysis of the causal map shows that Innovation influences positively Quality and Flexibility and it is positively influenced by Knowledge. As the Flexibility and Quality characteristics have a positive influence in other core-values, this means that if the level of Innovation increases in the network it is expected that the level of Flexibility, Quality, Customers' satisfaction, Reputation, and Employee's satisfaction will also increase.

By generating a new Organizations' core-values map that includes the RDC (as illustrated in Figure 7), it is possible to conclude that this potential member shares core-values with University A and B.

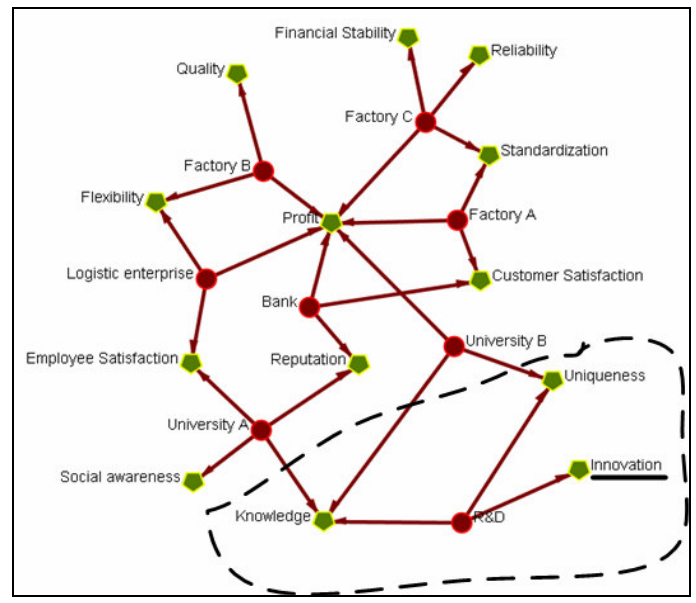

Figure 7 -Organization's core-values map after RDC joined the VBE.

In order to illustrate how to analyze the potential for conflicts among VO members and the alignment between VO's core-values and VO members' core-values, let us suppose that two business opportunities are identified by a broker, and a subset of these organizations are selected to form VOs.

In this case, the two following VOs were created:

- VO1 to develop a specific medicine. In this example VO1 selects Quality, Social awareness, Innovation, Uniqueness, and Profit as core-values.

- VO2 to manufacture pharmaceutical equipment. In this example VO2 selects Standardization, Customer satisfaction, and Profit as core-values.

When a VO is formed inside a VBE and assuming that the VO planner defines the set of core-values that will guide the behaviour of this VO, a VO's core-values map can be generated, which shows the cores values held by this VO. Since, various VOs can coexist at the same time in the context of a VBE, may be useful to analyze the shared values among VOs.

Figure 8 illustrates the core-values held by each VO. This map evidences that these two VOs give importance to distinct sets of core-values, and only the profit core-value is shared. 


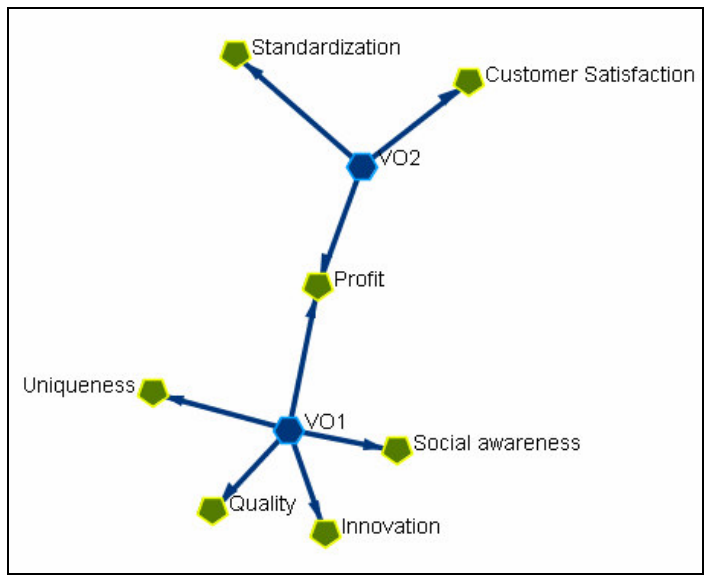

Figure 8 - VO's core-values map.

Since the purpose of VO2 is to manufacture pharmaceutical equipment, a factory that has the capacity to implement the specific manufacture process must be selected. Consequently the VO2 planner cannot choose for instance a Bank to do it, in spite of the Bank core-values having a higher level of alignment with VO's corevalues. Therefore, the partner selection cannot be made exclusively based on corevalues analysis; competencies fitness is naturally a must. However in the case that competences required are guaranteed, this analysis can be useful.
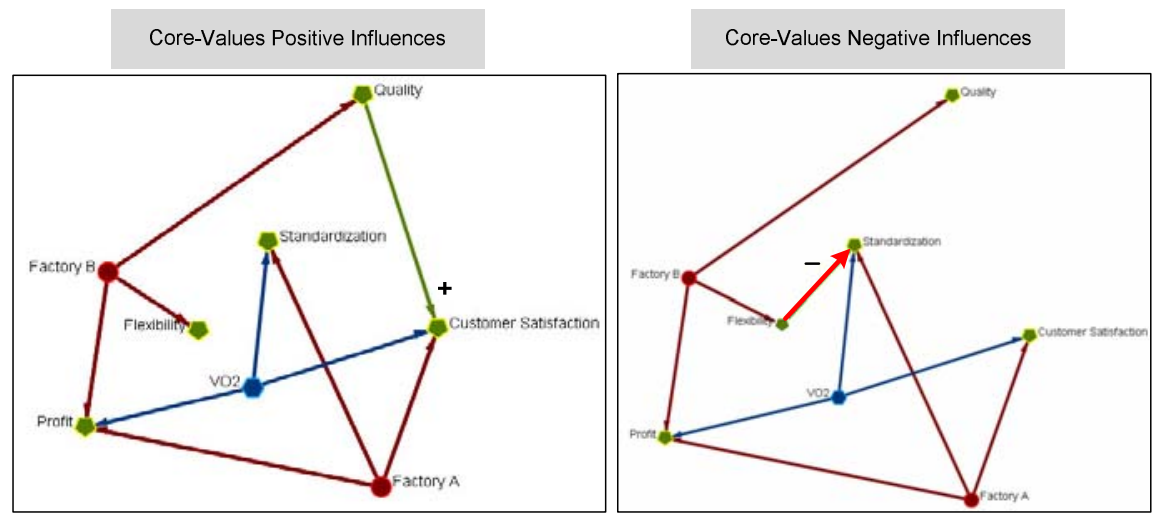

Figure 9-Alignment analysis between $\mathrm{VO} 2$ and potential members.

Based on the causal maps of Figure 9 it is possible to analyze the alignment of the VO2 with relation to Factory A and Factory B. Comparing these two potential members, it can be realized that Factory A's core-values fit better the VO2's corevalues than Factory B. Furthermore, the Flexibility core-value of Factory B has a negative influence on Standardization. As Standardization is a core-value of VO2, Factory B may have a negative impact in VO2's performance. 


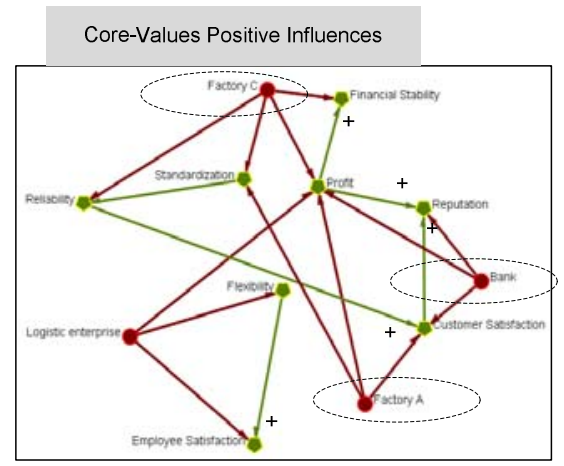

Core-Values Negative Influences

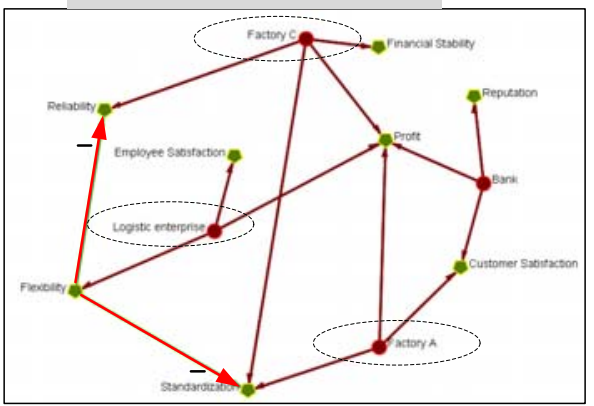

Figure 10 - Alignment analysis among VO2 members.

Let us, now suppose that VO2 is composed of the Bank, the Logistic Operator, the Factory A, and the Factory C. Based on Organization's core-value map for VO2, it is possible to analyze the potential for conflicts among VO members. As illustrated in

Figure 10, a potential for conflict between the Logistic Operator and Factory C can be detected. This potential for conflict is derived from the fact that the Flexibility core-value held by the Logistic Operator has a negative influence on reliability held by Factory C. Identical situation occurs in relation to Factory A and $\mathrm{C}$ due to the negative impact of Standardization on the Flexibility core-value.

It shall be noted that the above discussion is only an illustration based on the exemplified reference core-values. Different conclusions could naturally be derived from different causal maps.

\section{CONCLUSIONS}

Reaching a better characterization and understanding of the core-values role in collaborative processes is an important pre-condition to avoid conflicts and misunderstandings in the operation phase of VOs and VBEs. This understanding is also a base for the establishment of proper analysis methods to support decision making processes at various levels: VBE management, VBE membership, VO brokering, and VO planning.

The suggested approach proposes a model to analyze core-values alignment in a VBE context, inspired in the causal models and graph theories. The proposed model has the advantage of providing a visual/graphical representation which is easy to understand and to promote the communication between partners.

The applicability of the suggested approach was illustrated through the example presented; however the development of a full practical software tool to analyze the core-values in collaborative networks still requires further work.

Acknowledgments - This work as supported in part by the ECOLEAD integrated project funded by the European Commission. 


\section{REFERENCES}

1. Abreu A, Camarinha-Matos LM. "On the Role of Value Systems and Reciprocity in Collaborative Environments." In: Spring, ed. IFIP, Volume 224, Network-Centric Collaboration and Suporting Frameworks: Boston Springer, 2006.

2. Afsarmanesh H, Camarinha-Matos LM. "A framework for Management of Virtual Organization Breeding Environments". In: Collaborative Networks and their Breeding Environments: Springer, 2005: 35-48.

3. Barrett R. Building a Vison-Guided, Values-Driven organization, Paperback ed: ButterworthHeinemann, 2006.

4. Camarinha-Matos L, Macedo P. "Towards a Conceptual Model of Value Systems in Collaborative Networks". In: Establishing the Foundation of Collaborative Networks: Springer Boston, 2007: 5364.

5. Collins J, Porras J. Building your Company Vision. In: Havard Business Review, 1996.

6. Eden C. On the Nature of Cognitive Maps. Journal of Managemnet Studies 1992;29.

7. Greenland S, Brumback B. An overview of relations among causal modeling methods. International Journal of Epidemiology 2002.

8. Hall B. Values Shift: A Guide to Personal and Organizational Transformation: Resource Publications, 1995.

9. Higgins ET. An Experience that Creates Value. Journal of Cultural and Evolutionary Psychology ,Akadémiai Kiadó 2004;2:9-22.

10. Macedo P, Sapateiro C, Filipe J. "Distinct Approaches to Value Systems in Collaborative Networks Environments". In: Network-Centric Collaboration and Supporting Frameworks: Springer Boston, 2006: 111-120.

11. Rekom Jv, Riel CBMv, Wierenga B. A Methodology for Assessing Organizational Core Values. Journal of Management Studies 2006;43:175-201.

12. Rokeach M. The nature of human values. New York: Free Press. 1973.

13. Schwartz SH. Universals in the content and structure of values: Theoretical advances and empirical tests in 20 countries. Advances in experimental social psychology 1992:1-65.

14. Zineldin MA. Towards an ecological collaborative relationship management A "co-opetive" perspective. . European Journal of Marketing 1998;32:1138 - 1164. 